\section{SCALING WITH TOROIDAL CURRENT OF IMPURITY TRANSPORT IN ATC}

BY

E. S, MARMAR, S, A, COHEN, AND J, L, CECCHI

\section{PLASMA PHYSICS LABORATORY}

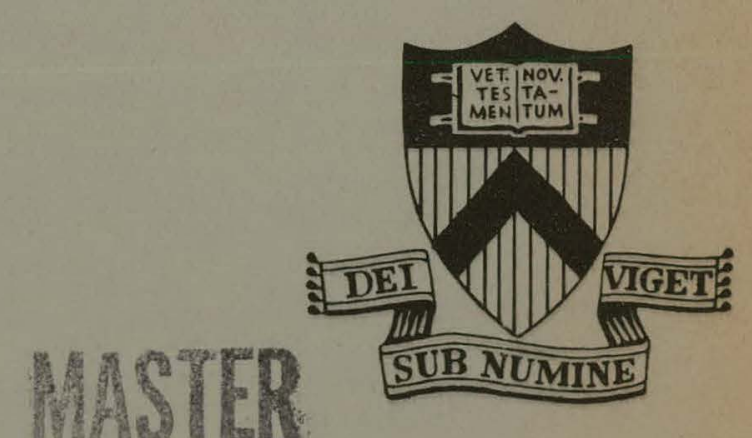

This work was supported by U. S. Energy Research and Development Administration Contract E(11-1)-3073. Reproduction, translation, publication, use and disposal, in whole or in part, by or for the United States Government is permitted. 


\section{DISCLAIMER}

This report was prepared as an account of work sponsored by an agency of the United States Government. Neither the United States Government nor any agency Thereof, nor any of their employees, makes any warranty, express or implied, or assumes any legal liability or responsibility for the accuracy, completeness, or usefulness of any information, apparatus, product, or process disclosed, or represents that its use would not infringe privately owned rights. Reference herein to any specific commercial product, process, or service by trade name, trademark, manufacturer, or otherwise does not necessarily constitute or imply its endorsement, recommendation, or favoring by the United States Government or any agency thereof. The views and opinions of authors expressed herein do not necessarily state or reflect those of the United States Government or any agency thereof. 


\section{DISCLAIMER}

Portions of this document may be illegible in electronic image products. Images are produced from the best available original document. 
This report was prepared as an account of work sponsored by the United States Government. Neither the United States nor the United States Energy Research and Development Administration, nor any of their employees, nor any of their contractors, subcontractors, or their employees, makes any warranty, express

or implied, or assumes any legal liability or responsibility for the accuracy, completeness or usefulness of any information, apparatus, product or process disclosed, or represents that its use would not infringe privately owned rights.

Printed in the United States of America. Available from

National Technical Information Service

U. S. Department of Commerce 5285 Port Royal Road

Springfield, Virginia 22151

Price: Printed Copy $\$ \ldots$; Microfiche $\$ 3.00$

*Pages

$1-50$

$51-150$

$151-325$

$326-500$

501-1000
NTIS

Selling Price

$\$ 4.00$

5.45

7.60

10.60

13.60 


\section{SCALING WITH TOROIDAL CURRENT OF IMPURITY \\ TRANSPORT IN ATC}

E. S. MARMAR, S. A. COHEN, J. L. CECCHI

Plasma Physics Laboratory, Princeton University

Princeton, New Jersey, U.S.A.

\section{ABSTRACT}

We report on an experiment measuring the scaling with changing plasma conditions of a parameter characteristic of the transport of aluminum injected into the ATC tokamak. This parameter is the time after injection at which the photon signal from $A 1 X I$ reaches its maximum. It is found that the data are in agreement with the predictions of a computer code which uses neoclassical theory in the Pfirsch-Schlüter regime. An approximate model describing impurity transport in ATC is also presented. This model is utilized to calculate the expected scaling with current of the aluminum transport assuming classical, neoclassical and pseudoclassical forms for the diffusion coefficient. The data are in agreement with both the neoclassical and pseudoclassical results from this model:

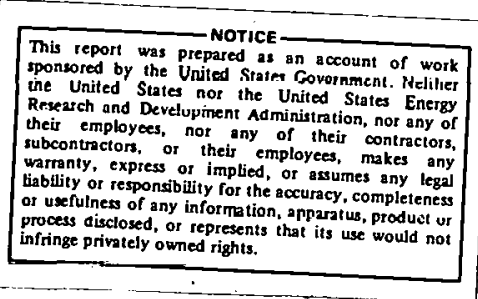




\section{INTRODUCTION}

Employing laser-impelled impurity injection [1], in conjunction with VUV spectroscopy, we have previously measured and reported on the transport of aluminum ions in the ATC tokamak [2]. The results showed good agreement with the predictions of neoclassical theory in the Pfirsch-Schlüter regime. To pursue further the question of whether heavy impurity transport is indeed neoclassical, we have performed an experiment to measure the scaling with changing plàsma conditions of a particular parameter characteristic of that transport.

As described elsewhere [1], the impurity injector provides a delta function-like source in time of neutral Al atoms at the edge of the piasma. The $A l$ is quickly ionized and transported along the toroidal field lines; higher states of ionization appear successively as the ions are more slowly transported across the field into the hotter, denser region of the plasma. Using a grazing incidence VUV monochromator, we observe the line integral of photon signals in the midplane across the minor cross section. Typical signals for transitions of Al VII-AI XI are shown in Fig. 1. A parameter characteristic of the transport rate is the time (after injection) at which the signal from a given Al ionization state reaches its maximum. The peak time for the Al XI signal, $t_{X I}$, has been measured for Al injection into hydrogen discharges whose toroidal current $I_{p}$ was varied from 40 to $80 \mathrm{kA}$. Al XI was chosen because it is the highest observable aluminum ionization statc in the VUV, since the He-like Al XII and H-like AI XIII 
both radiate only weakly. The Al XI appears after the greatest radial excursion from the edge of the plasma, peaking typically at $r=10 \mathrm{~cm} \mathrm{[2],} \mathrm{and} \mathrm{is} \mathrm{thus} \mathrm{the} \mathrm{most} \mathrm{sensitive} \mathrm{to} \mathrm{changing}$ transport rates. Radial profiles of impurities, in addition to being tedious and difficult to measure due to the need. for Abel inversions,: are relatively insensitive to the transport rates, the position of a particular ionization state depending mainly on the electron temperature profile. (Profile widths are useful for distinguishing spreading plus inward transport from the case of spreading transport alonel. The variation of $t_{X I}$ with plasma current, density--and-temperature has been caiculated using a- - . computer code based on neoclassical theory. The results are in agreement with the experiment. A simplified model for predicting $t_{X I}$ is developed to facilitate the computation and to elucidate the physical processes-involved-in the-transport. This modelallows convenient calculation of $t_{\mathrm{XI}}$ using various forms for the diffusion coefficient. We have performed such computations for classical, neoclassical and pseudoclassical coefficients, and find that both the neoclassical and the pseudoclassical scale in agreement with the data.

\section{EXPERIMENTAL RESULTS AND COMPARISON WITH THEORY}

Figure 2 shows the time dependence of electron density and current for the case of a $60 \mathrm{kA}$ : (peak current) ATC discharge. The density is measured by a $4 \mathrm{~mm}$ microwave interferometer system and the current by a Rogowski loop. The Al was injected at $t=25$ ms, just at the beginning of the flat top in the plasma current. 
Figure 3 shows the data points for $t_{X I}$ vs. current. The data are compared to the results of a computer code which integrates the coupled diffusion equations including ionization and recombination source terms:

$$
\frac{\partial n_{j}}{\partial t}=-\frac{1}{r} \frac{\partial}{\partial r}\left(r \Gamma_{j}\right)+s_{j} \quad j=1, \ldots 13,
$$

where $r$ is the minor radius and the $s_{j}$ are the ionization [3] and recombination [4] source terms to the $j^{\text {th }}$ ionization state: The flux of the $j^{\text {th }}$ species is given by:

$$
\bar{\Gamma}_{j}=\sum_{b} \frac{\rho_{b}^{2} z_{b} v_{b j}}{z_{j} T_{b}}\left(i+\alpha_{b} q^{2}\right)\left(T_{b} \frac{\partial n_{b}}{\partial r}-\frac{n_{b}}{n_{j}} \frac{z_{b}}{z_{j}} T_{j} \frac{\partial n_{j}}{\partial r}\right),
$$

where the collision frequency has the form

$$
v \nu_{b j}=\frac{4 \sqrt{2 \pi} n_{j} z_{j}^{2} z_{b}^{2} e^{4} \ln \Lambda}{3 m_{b}{ }^{1 / 2} \mathrm{~T}_{b} 3 / 2}
$$

$\mathrm{n}_{\mathrm{b}}, \mathrm{m}_{\mathrm{b}}, \mathrm{T}_{\mathrm{b}}, \mathrm{z}_{\mathrm{b}}$, and $\rho_{\mathrm{b}}$ are the density, mass, temperature, chäge, and gyro-radius of the $b^{\text {th }}$ background species; $n_{j}, T_{j}$, and $z_{j}$ are the density, temperature, and charge of the $j^{\text {th }}$ state of $A l ; q$ is the safety factor and the $\alpha_{b}$ are constants of order unity. Equation 2 is obtained by extending the theory of Rutherford [5] to the case of a (negligibly) small amount of Al diffusing in a background plasma containing more than one ion species: We have calculated that diffusion caused by collisions between different Al ionization states, as well as that caused by temperature gradient terms, is negligible. In the present calculation, the background plasma is assumed to be stationary in time and composed of hydrogen and oxygen, with the ratio of oxygen to electron 
densities determined from an estimated $\mathrm{z}_{\text {eff }}=5$ on axis. The electron density and oxygen density and temperature profiles are taken as parabolic, and the electron temperature profile is taken as the square of a parabola [6]. The charge profile of the oxygen is then approximated, utilizing experimental observations of OVI and OVII [7], by

$$
\bar{z}_{0}(r)=4+4\left(1-r^{2} / a^{2}\right)^{1.84}
$$

Charge neutrality then determines the proton density profile. In "standard" tokamak discharges, the term proportional to $\partial \mathrm{n}_{\mathrm{b}} / \partial \mathrm{r}$ causes inward impurity transport, while the term propor: tional to $\partial n_{j} / \partial r$ causes spreading of the impurity profile. Using $x$, the electron impact excitation coefficient given by [4]

$$
\mathrm{X}=\frac{1.6 \times 10^{-5} \mathrm{f}_{\mathrm{pq}}}{\Delta \mathrm{E} \mathrm{T}_{\mathrm{e}}^{1 / 2}}\langle\overline{\mathrm{g}}\rangle \mathrm{e}^{-\Delta \mathrm{E} / \mathrm{T}_{\mathrm{e}}}
$$

where $f_{p g}$ is the absorption oscillator strength for the transition from the state $g$ to the state $p, \bar{g}$ is the Gaunt factor averaged for a Maxwellian electron distribution, $\Delta \mathrm{E}$ is the transition energy in $\mathrm{eV}$ and $\mathrm{T}_{\mathrm{e}}$ is the electron temperature in $\mathrm{eV}$, the monochromator signal $\mathrm{B}_{\mathrm{XI}}$ is given by

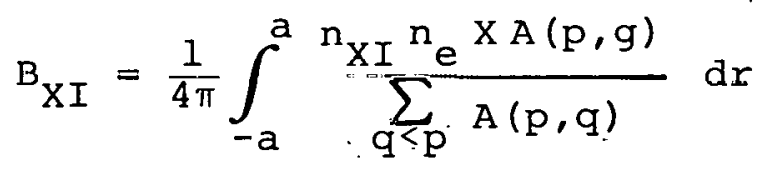

where $n_{X I}$ is the density of $A I X I$, and $A(p, q)$ is the spontaneous probability for transition between the states $p$ and $q$ ( $g$ is the ground state), and the integration is along the line of sight of the monochromator. Thus $t_{X I}$ can be predicted. 
When the plasma current is varied, while the toroidal field is held fixed, $\mathrm{T}_{\mathrm{e}}(\mathrm{r})$ and $\mathrm{n}_{\mathrm{e}}(\mathrm{r})$ also change. The variation of the average density was measured with the microwave system. However, as Thomson scattering scans were not available during the experiment, we are forced to assume some scaling for $T_{e}$ with $I_{p}$. The results from a large number of Thomson scattering scans on ATC [6] indicate that $\mathrm{T}_{\mathrm{e}}{ }^{\alpha . I_{\mathrm{p}}}{ }^{1 / 2}$ and we have chosen to use this scaling. The implications of this assumption will be discussed in sect. 4 . We have also assumed that $\mathrm{Z}_{\text {eff }}$ and all profile shapes are indepen dent of current. The results of the computer calculations are shown as the open circles in Fig. 3, and, within the experimental shot-to-shot deviation, are in excellent agreement with the data.

\section{APPROXIMATE MODEL}

To elucidate the competing effects which contribute to the variation of $t_{X I}$ with current, as well as to facilitate comparison with other possible transport theories, we present an approximate model for calculating $t_{X I}$. Consider, as in Fig: 4, that the impurities are in a shell-like radial distribution, and the peak of the density moves inward at some velocity $v(r)$. As time proceeds, the Al ionizes to successively higher states. For an electron density $\mathrm{n}_{\mathrm{e}}$ and ionization coefficient $\mathrm{s}_{\mathrm{a} \rightarrow \mathrm{b}}$, the rate at which ionization from state a to state b takes place is $n_{e} s_{a \rightarrow b}$. Consider a particular ionization state and integrate $\mathrm{n}_{\mathrm{e}} \mathrm{S}_{\mathrm{a} \rightarrow \mathrm{b}}$ over time as the ions diffuse inward. The peak time of the photon signal for state $b$ should correspond to the limit of integration where the integral becomes equal to a constant of order unity. This integral condition for $t_{X I}$ is expressed as 


$$
\int_{0}^{t} n_{e} s_{X \rightarrow X I} d t^{\prime}=c,
$$

where $C \approx 1$ and is determined exactly from a comparison to the full code. The integrand depends upon the radial location $r$ of the density peak of the diffusing species. Therefore, $\mathrm{n}_{e}$ and $S_{X \rightarrow X I}$ depend upon $t$ through the equation of motion

$$
\frac{d r}{d t}=v(r)
$$

with the initial condition $r(t=0)=r_{0}, \dot{r}_{0}$ being the injection deposition radius $[1] \cdot \because$ Taking the lower limit in Eq. 6 to be :. zero is an approximation. This approximation is valid because $S_{X \rightarrow X I}$ is negligibly small for the times when the peak of the Al distribution is at radii larger than $r\left(t_{x}\right)$.

To make this..condition useful, -we require a convenient ...... expression for $\mathrm{V}(\mathrm{r}) \div$ Since the total Al-flux is equal. to the -... product of total Al density and $v(r)$, we write.

$$
v(r)=\left(\sum_{j} \Gamma_{j}\right) /\left(\sum_{j} n_{j}\right)
$$

Specializing to neoclassical diffusion, we obtain from Eqs: 2,3, and 9

$$
\begin{array}{r}
v(r)=\frac{1}{N} \sum_{j} \cdot \sum_{b}\left\{\frac{4 \sqrt{2 \pi}}{3} \frac{e^{4} \ln \Lambda}{m_{b}^{1 / 2} T_{b}^{3 / 2} \rho_{b}^{2} z_{b}^{3}\left(1+\alpha_{b} q^{2}\right)}\right. \\
\quad x\left(n_{j} z_{j} \frac{\partial n_{b}}{\partial r}-n_{b} z_{b} \frac{\left.\partial n_{j}\right)}{\partial r}\right\}
\end{array}
$$

where $\mathrm{N} \equiv \sum_{j}\left(\mathrm{n}_{j}\right)$ is the total $\mathrm{Al}$ - density and we have assumed that $\mathrm{T}_{\mathrm{j}}=\mathrm{T}_{\mathrm{b}}$ for $\mathrm{all} \mathrm{b}$ and $\mathrm{j}$. The first simplifying assumption we will 
make is to eliminate the sum over b by considering only the oxygen terms (since their effect is greater than that of the protons), and we utilize the charge profile for 0 given by Eq. 4. Summing over $j$ results in the factor $\overline{\mathrm{Z}}_{\mathrm{Al}}$ given by.

$$
\bar{z}_{A l}=\frac{1}{N} \sum_{j} n_{j} z_{j}
$$

multiplying the $\frac{\partial \mathrm{n}_{\mathrm{b}}}{\partial \mathrm{r}}$ term. $\quad \overline{\mathrm{z}}_{\mathrm{Al}}$ can be approximated by an analytical expression explicitly involving $t$ which is unity at $t=0$ and gives $\bar{Z}_{A I}=11$ as $t: \rightarrow \infty$, with a parameter $t_{\max }$ chosen to match the full code for the $60 \mathrm{kA}$ case: $\bar{z}_{A l}(t=3.23)=10$ (see Fig. $\left.5(a).\right)$ This expression is

$$
\overline{\mathrm{z}}_{\mathrm{Al}}=1+10\left[1-\exp \left(-t / t_{\max }\right)\right] .
$$

The sum over $j$ for the $\frac{\partial n_{j}}{\partial r}$ term results in a logarithmic derivative of $\mathrm{N}$ which we approximate by

$$
\frac{1}{N} \sum_{j} \frac{\partial n_{j}}{\partial r}=\frac{1}{N} \frac{\partial N}{\partial r} \approx \frac{1}{(a-r)}
$$

where $a$ is the plasma minor radius. The spreading term is included in $\mathrm{V}(r)$ because spreading diffusion coupled with the asymmetric loss of particles at the edge of the plasma leads to a net inward movement of the peak of the total impurity density distribution.

Incorporating all of these approximations, we put Eq. 10 into a form which makes the terms with radial dependence explicit

$$
v(r)=\frac{\gamma\left(I+\alpha_{O} q^{2}\right)}{T_{0}^{1 / 2}}\left[\bar{z}_{0} \bar{z}_{A l} \frac{\partial n_{O}}{\partial r}+\frac{{\overline{z_{O}}}^{2} \cdot n_{O}}{(a-r)}\right]
$$




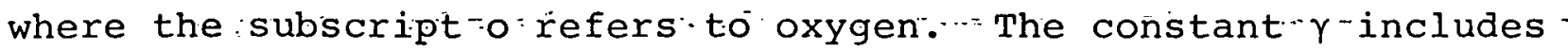
all the factors in Eq. 10 not appearing explicitly in Eq. 14, plus a free multiplicative constant to be determined along with $\mathrm{C}$ of Eq. 6 by simultaneously matching, for the $60 \mathrm{kA}$ case, the model $t_{X I}$ ant $r\left(t_{X I}\right)$ with the full code peak time and the radius of maximum $A l$ density at the time respectively. Figure $5(b)$ shows the comparison between $v(r)$ from the full code and the approximate model. It is found that the free parameter in $\gamma$ equals 1.1, and $C=2: 14$. Having determined $C$ and $\gamma$, they are then held fixed while $I_{\dot{p}}, T_{e}{ }_{-}$and $n_{e}$ are varied $:-F i g u r e-6$ shows the comparison of the results of the approximate theory with those of :the ful1-:code for two : $t_{X}$ is made-wi-th the approximate-theory by simply replacing $\ldots$ $\mathrm{S}_{\mathrm{X} \rightarrow \mathrm{XI}}$ with $\mathrm{S}_{\mathrm{IX} \rightarrow \mathrm{X}}$ in $\mathrm{Eq} \cdot 6:$

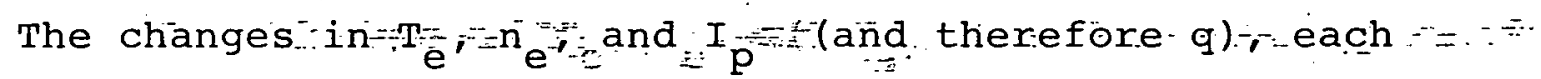

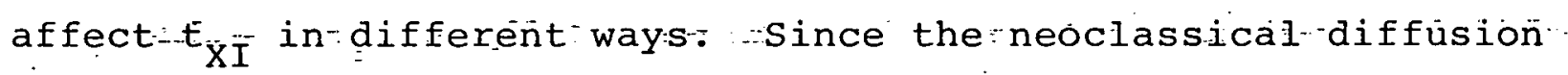
coefficient is.proportional: to $\left(1+\alpha q^{2}\right)$, increasing the current decreases this factor and $t_{X I}$ should get longer. However, increasing $I_{p}$ increases $T_{e}$ ' and $n_{e}$, and thus the ionization rate at a given radius. This shortens the radial distance the peak mast move to satisfy $\because \mathrm{Eq} \doteqdot \sigma$; and sincé this latter effect dominates, $t_{X I}$ is:shortened... Both the: transport:and the ionization are. linear in $\mathrm{n}_{\mathrm{e}} \cdot$. If $\mathrm{n}_{\mathrm{e}}$ were the only background plasma parameter........

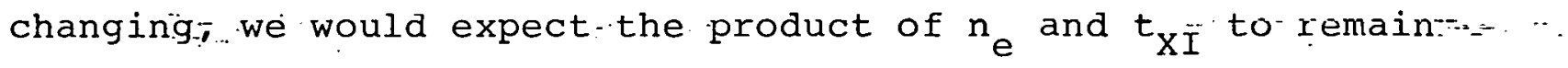
constant $\mathrm{T}_{\mathrm{b}}$ also appears - in - the transport coefficient:-

$$
v(r) \propto \frac{1}{T_{b} 1 / 2}
$$


The scaling of Artsimovitch [8] predicts a relatively weak dependence of $T_{b}$ on $I_{p}: T_{b} \alpha \cdot\left(n_{e} I_{p}\right)^{1 / 3}$. The diffusion coefficient would then vary as $\left(n_{e} I_{p}\right)^{-1 / 6}$. We have not included any effects from changing $\mathrm{T}_{\mathrm{b}}$ in these results.

Different transport theories can be compared with the neoclassical using Eqs. 6 and 7, with a different form for $v(r)$, and thus different dependences on plasma parameters. For the plasma conditions in this experiment, the effective collision frequency $\nu^{*}=\nu R_{0} \cdot q /\left(\varepsilon^{3 / 2} v_{T}\right)$ [9] for aluminum-oxygen collisions at $r=10 \mathrm{~cm}$ is approximately equal to 230 , so $\nu^{*} \varepsilon^{3 / 2}=20$, and we expect to be well into the Pfirsch-Schlüter regime. If the transport were caused by some kind of plasma turbulence (for example drift waves) one might expect the diffusion coefficient to have the pseudoclassical form [10]:

$$
D_{p c}=\frac{\nu_{c}}{\omega_{e}}\left(\frac{C T \cdot e}{e B}\right)\left(\frac{\tilde{n}}{n}\right)^{2} \frac{k_{i}^{2}}{k_{n}^{2}}
$$

where $v_{C}$ is the electron-ion collision frequency, $w_{e}$ the electron gyrofrequency, $\tilde{n} / \mathrm{n}$ the level of density fluctuations, $\mathrm{k}_{\perp}$ the perpendicular wavevector of the fluctuations $\approx 1 / \rho_{i}[10]$, and $k_{i}$ is.the parallel wavevector of the fluctuations $\approx 1 / R_{0} q$. [11].

In Fig. 7 we show the results form Eqs. 6 and 7 for the two transport theories, clașsical and pseudö-classical and compare them with the neoclassical. We take the following forms for the respective diffusional velocities:

$$
v_{\text {classical }}=\gamma_{c} \frac{n_{b}}{T_{b}^{1 / 2}}\left[\frac{1}{n_{b}} \bar{z}_{b} \bar{z}_{j} \frac{\partial n_{b}}{\partial r}+\frac{\overline{\bar{z}}_{b}^{2}}{(a-r)}\right]
$$




$$
v_{\text {pseudo-classical }}=\frac{\mathrm{n}_{\mathrm{b}} \overline{\bar{z}}_{\mathrm{b}}^{4} \mathrm{q}^{2}\left(\frac{\tilde{\mathrm{n}}}{\mathrm{n}}\right)^{2}}{\mathrm{~T}_{\mathrm{e}}^{1 / 2} \mathrm{~T}_{\mathrm{b}}}\left[\frac{1}{(\mathrm{a}-\mathrm{r})}\right] \begin{aligned}
& \text { ('spreading only } \\
& \text { no inward term) }
\end{aligned}
$$

The velocities are normalized, through the choice of $\gamma_{C}$ for the classical and $\tilde{n} / n$ for the pseudoclassical, to make $t_{X I}$ equal

$3.23 \mathrm{~ms}$, for the $60 \mathrm{kA}$ discharge. Without this normalization, the classical transport is more than a factor of 10 too slow. The motivation for including the classical calculation is to show explicitly the effect of the $\mathrm{q}^{2}$ term in the diffsuional flux. For the pseudo-classical theory, it is assumed that $\tilde{\mathrm{n}} / \mathrm{n}$ is constant, independent of radius and plasma current. Since both the PfirschSchlüter and pseudo-classical coefficients are approximately proportional to $\mathrm{q}^{2}$, it is not surprising that they scale almost identically in this model. The pseudo-classical transport requires $\tilde{n} / \mathrm{n}$ of about $10^{-2}$ for the observed transport. This is to be compared with experimental observations of small scale electron density fluctuations in ATC which have been performed.with microwave [11] and $\mathrm{CO}_{2}$ laser [12] scattering. Estimates of $\tilde{\mathrm{n}} / \mathrm{n}$ from these two methods are $\gtrsim 5 \times 10^{-3}$ and $1 \times 10^{-2}$ respectively. On the basis of thïs scaling of $t_{X I}$ with $I_{p}$, as well as the absolute magnitude of $t_{X I}$, it is not possible to distinguish between neoclassical and pseudo-classical transport.

\section{IMPLICATIONS OF UNKNOWN PROFILES}

As previously mentioned, certain parameters of the plasma were not measured during the course of this experiment. The most 
important of these are the electron temperature and density profiles (average electron density is. known). Using the approximate model, we compare the predictions for $t_{X I}$ with various assumptions for these profiles. Figure 8 (a) shows the results for the three choices :

$$
\mathrm{T}_{\mathrm{e}} \propto \mathrm{I}_{\mathrm{p}}^{\varepsilon}\left(1-\mathrm{r}^{2} / \mathrm{a}^{2}\right)^{2} \quad \varepsilon=.3, .5 \text { and } .7 \text {, }
$$

where $I_{p}$ is the plasma current, $r$ the minor radius, and a the limiter radius. Figure $8(\mathrm{~b})$ shows the comparisons for:

$$
T_{e} \propto I_{p}^{-1 / 2}\left(1=r^{2} / a^{2}\right) n . \quad n=1,5,2 \text { and } 3,
$$

and finally Fig..8(c) compares ty for the three cases:

$$
\mathrm{n}_{\mathrm{e}}=\mathrm{n}_{\mathrm{e}_{\mathrm{o}}}\left(1-\mathrm{r}^{2} / \mathrm{a}^{2}\right)^{\xi}, \quad \xi=.5,1 \text { and } 1.5
$$

Once again all 1 curves have been, normalized to $=3-2-3$ msec for thess $=$ : $60 \mathrm{kA}$ case. It -is" clear.from these three graphs, that "even over the wide-variation allowed for the parameters $\varepsilon, n$, and $\xi$, the àgreement of the predictions with the experiment is still good.

\section{CONCLUSIONS}

We have performed an experiment to measure the scaling of the peak time: nf the Al:XI photon signal with changing plasma conditions - - The-results are-in-agreement with the predictions of both neoclassical theory in the Pfirsch-Schlüter regime, and pseudoclassical transport with $\tilde{n} / n \approx 10^{-2}$. 
ACKNOWLEDGEMENTS

This work was. supported by the U. S. Energy Research and Development Administration Contract No: E(11-1)-3073. 
REFERENCES

[1] MARMAR, E. S., CECCHİ, J. L., COHEN, S. A., Rev. Sci. Instrum. $\underline{46}$, 1149 (1975).

[2] COHEN, S. A., CECCHI, J. L., MARMAR, E. S., Phys. Rev. Lett. 35,1507 (1975).

[3] LOTZ, W., Astrophys. J. Suppl. Ser. 14, 207 (1967).

[4] ELTON, R. C., in Methods of Experimental, Physics, edited by H. R. Griem and R. H. Lovberg (Academic, New York, 1970), Vol. 9, Pt. A.

[5] RUTHERFORD, P. H., Phys. Fluids 17, 1893 (1974).

[6]. DAUGHNEY; C. C., Nucl. Fus. 15, 967 (1975).

[7] CECCHI, J. L., Princeton Plasma Physics Lab. Report MATT-1256 (1976), to be published.

[8] ARTSImOVItCH, L. A., Nucl. Fus. 12, 215 (1972).

[9] FURTh, H. P., Nucl. Fus. 15, 487 (1975).

[10] YOSHIKAWA, S., Phys. Fluids 16, 1749 (1973).

[11] Mazzucato, E., Phys. Rev. Lett. $\underline{36}, 792$ (1976).

[12] SLUSHER, R. E., SURKO, C. M., PORKOLAB, M., CECCHI, J. L., Bull. Am. Phys. SOC. 21,848 (1976): 


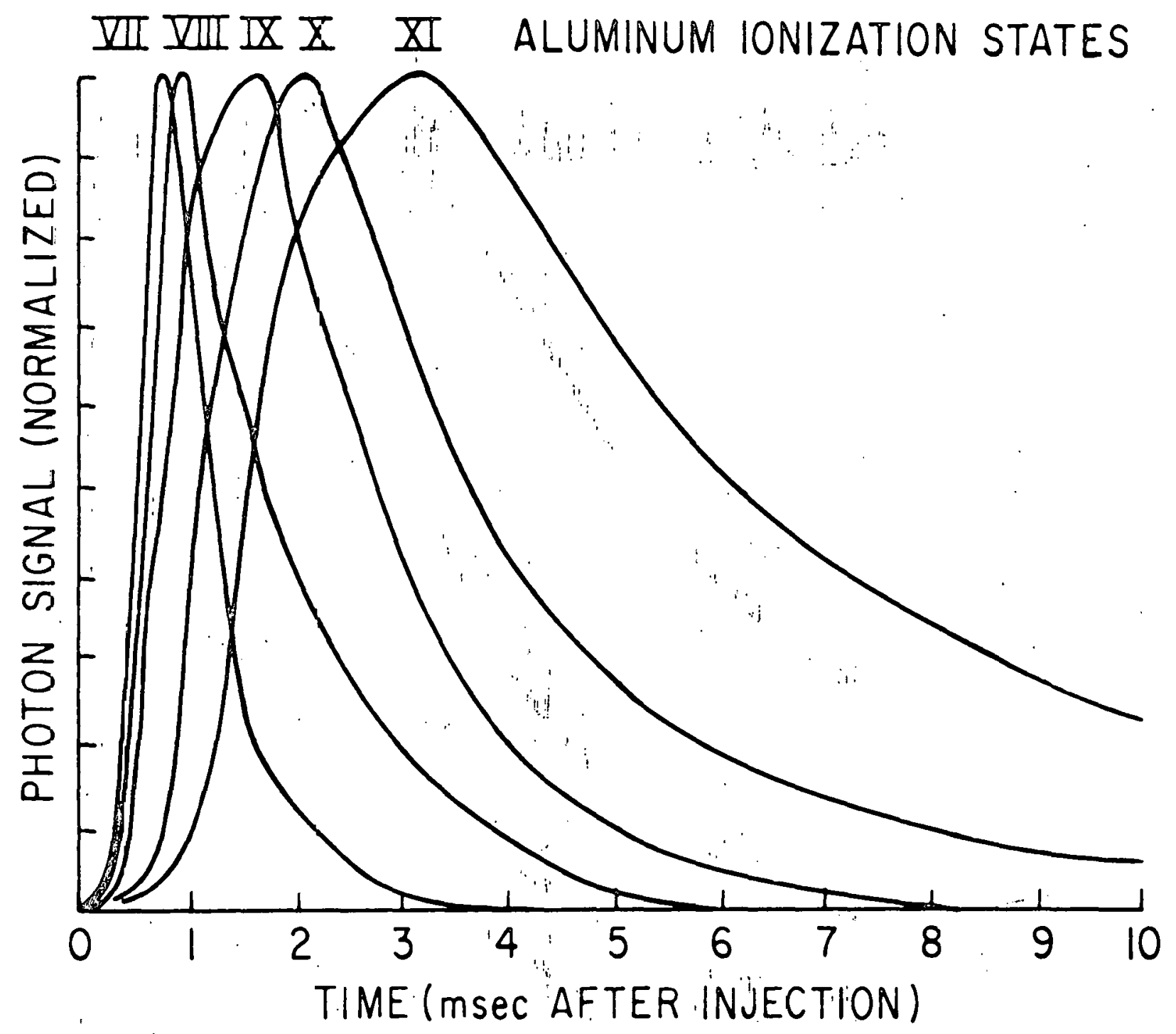

763143

Fig. 1. Time evolutions of experimental line integral photon signals after aluminum injection for transitions of. Al VII througia AI XI. 


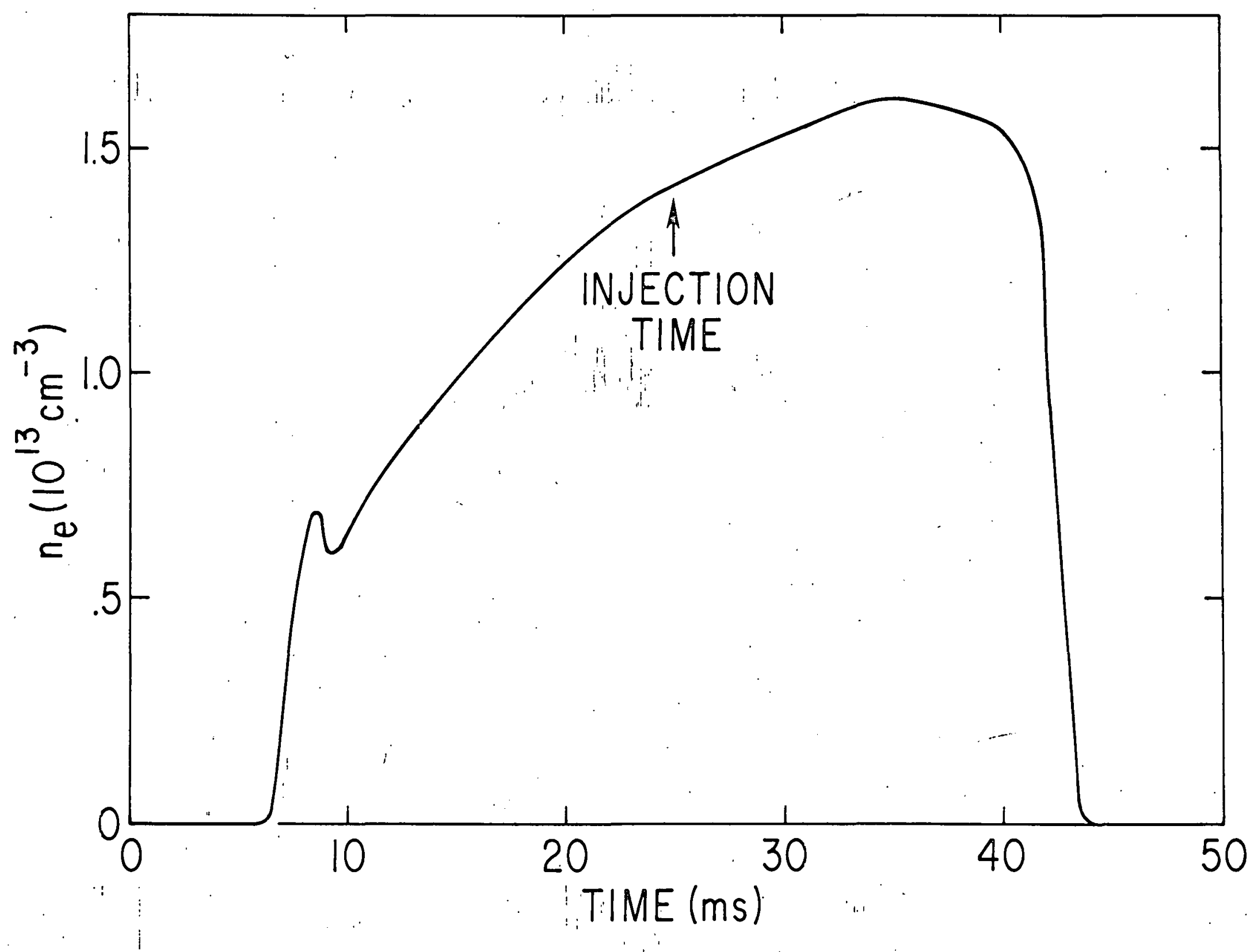
radiaig. 2. (a) Peak electron density (assuming a parabolic 


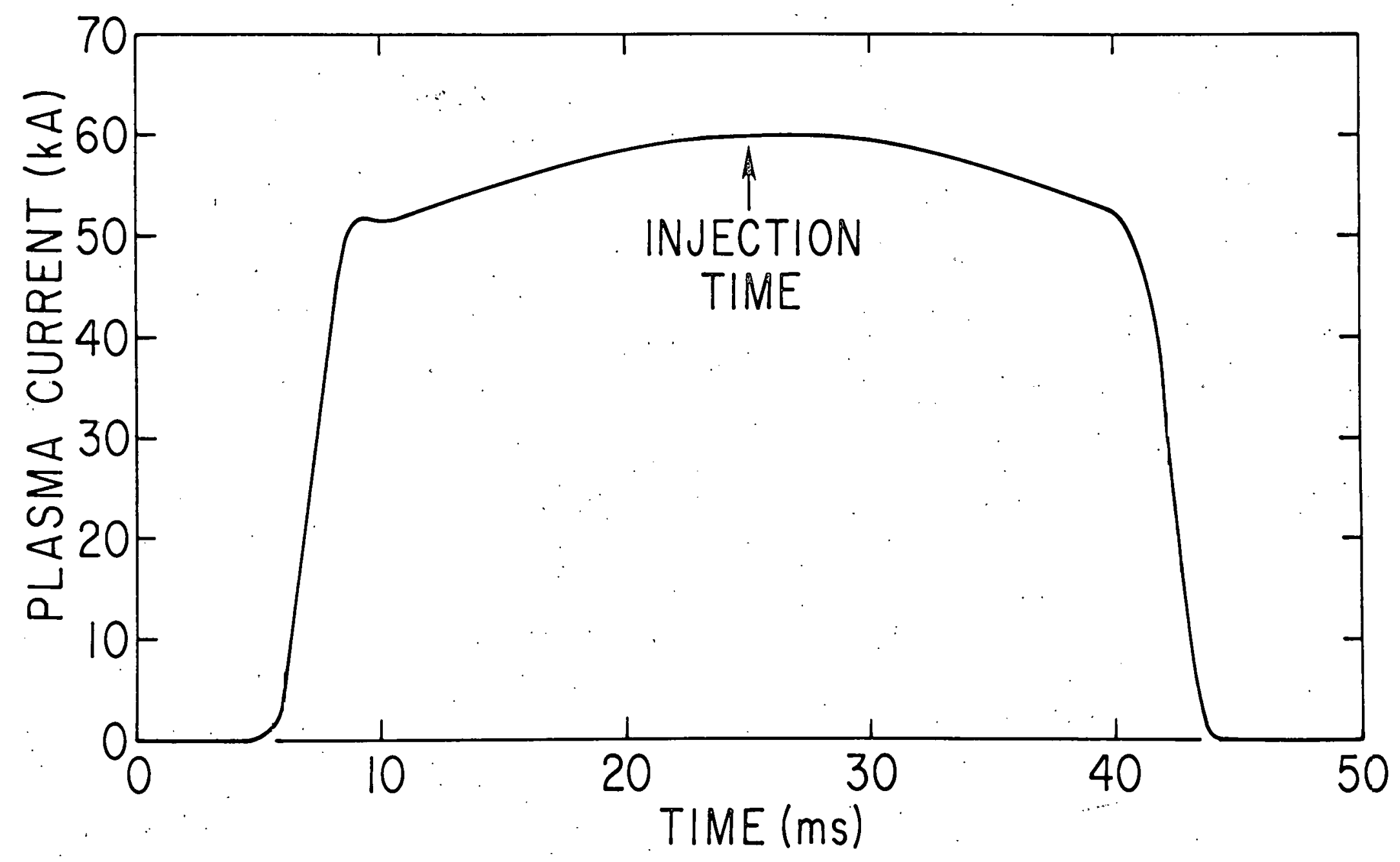

Fig. 2. (b) Toroidal plasma current for the $60 \mathrm{kA}$ peak current discharge. 


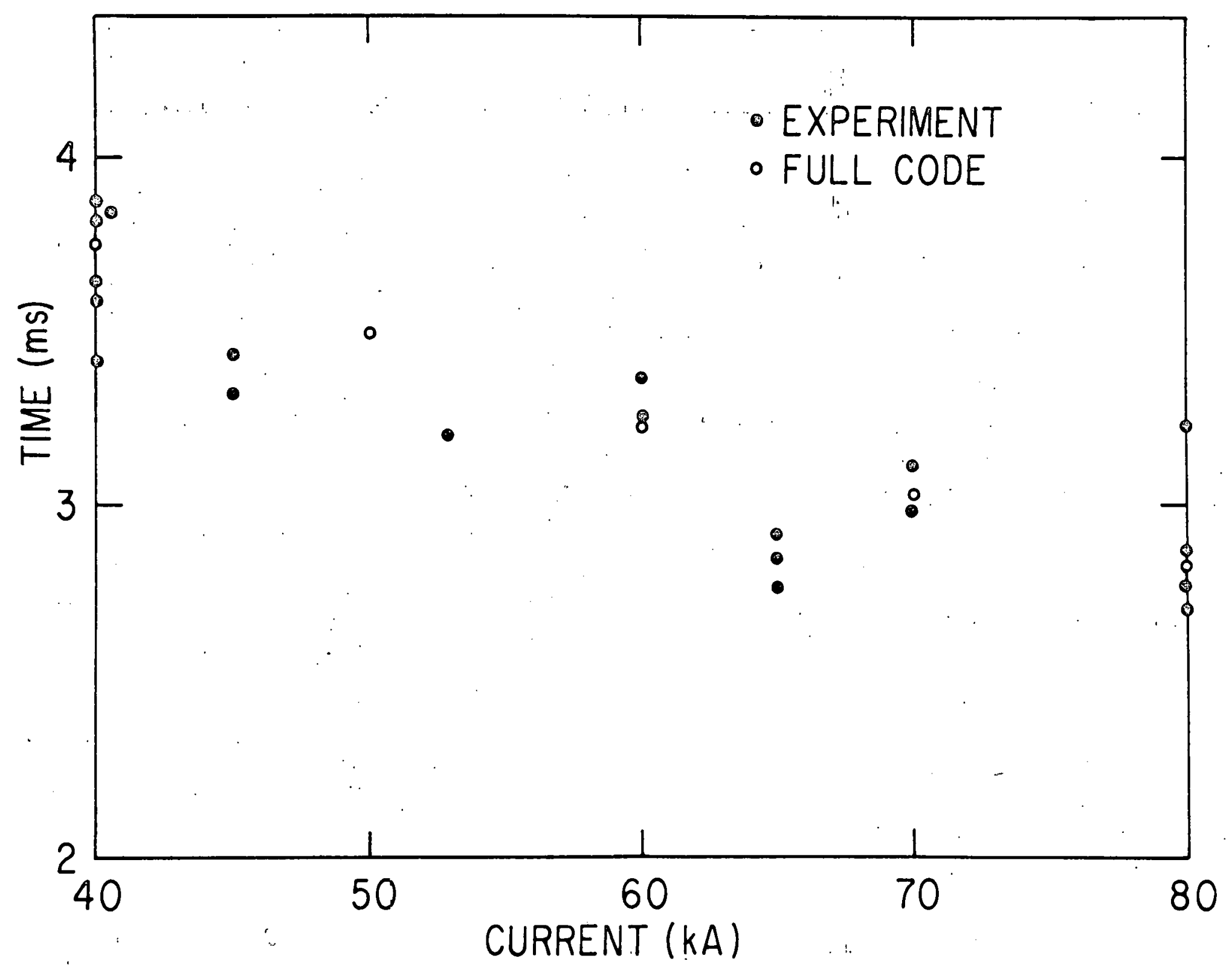




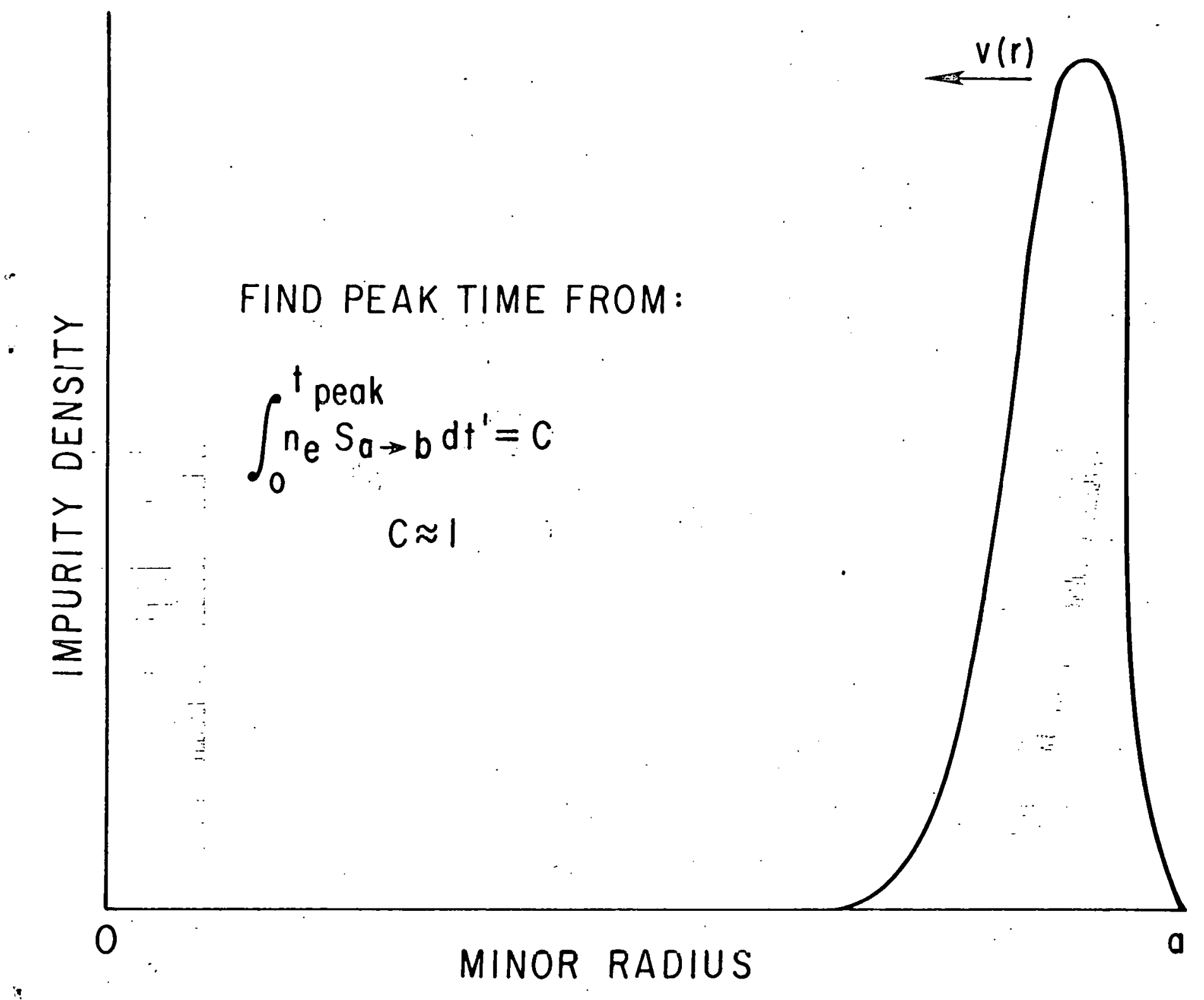

$\Leftrightarrow$

763496

Fig. 4. Approximate model used to predict tXI for various. diffusion $=$ coefficients $n_{e} S_{a} \rightarrow b$ is the-electron impact-ionization ratee from-state a-to-state $b$ and $v(r)=\Gamma_{D} / N_{\text {impurity: }}$ 


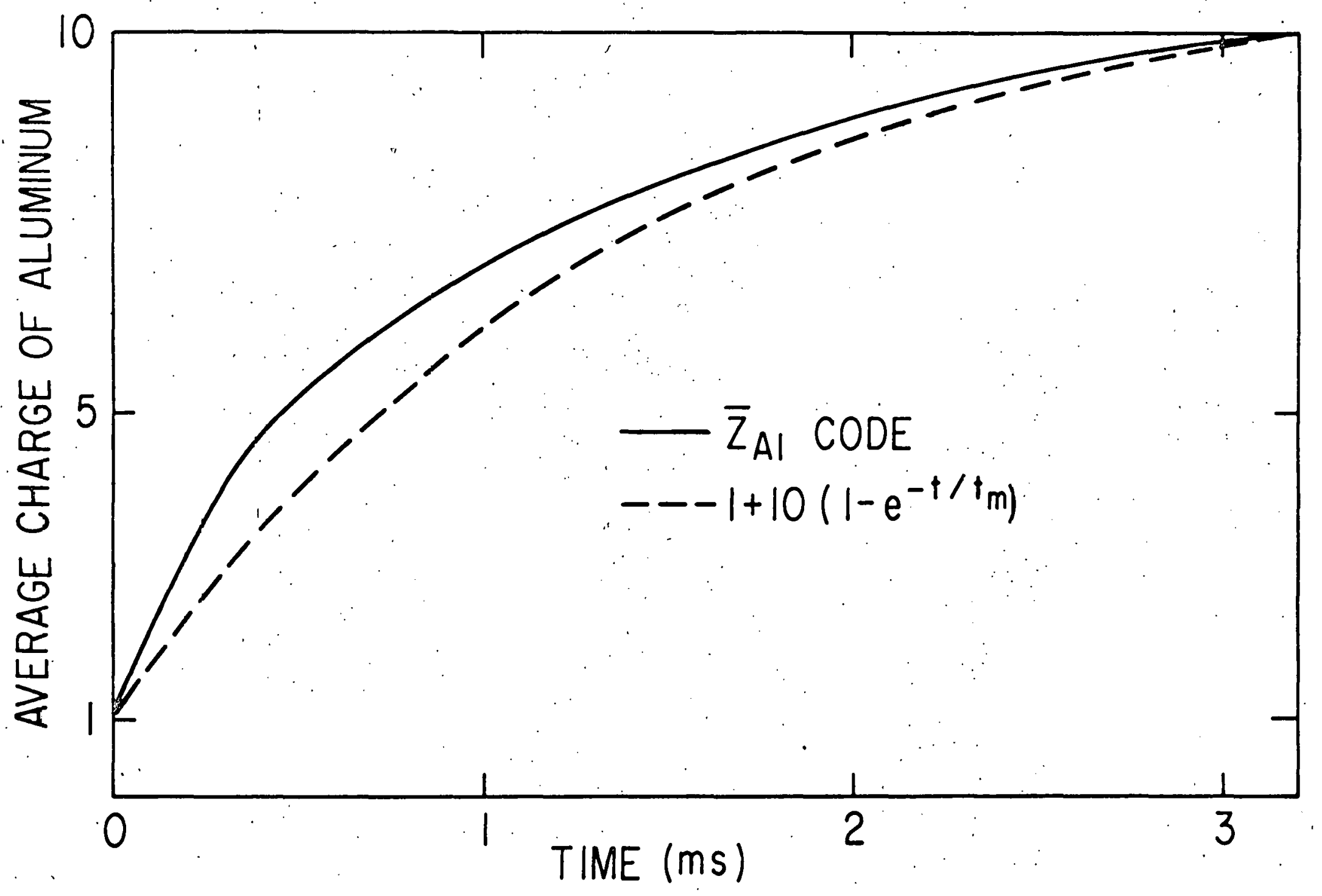

763459

Fig. 5. (a) Comparison of $\bar{z}_{A l}$ from the full code with the formula used in the approximate model calculations. 


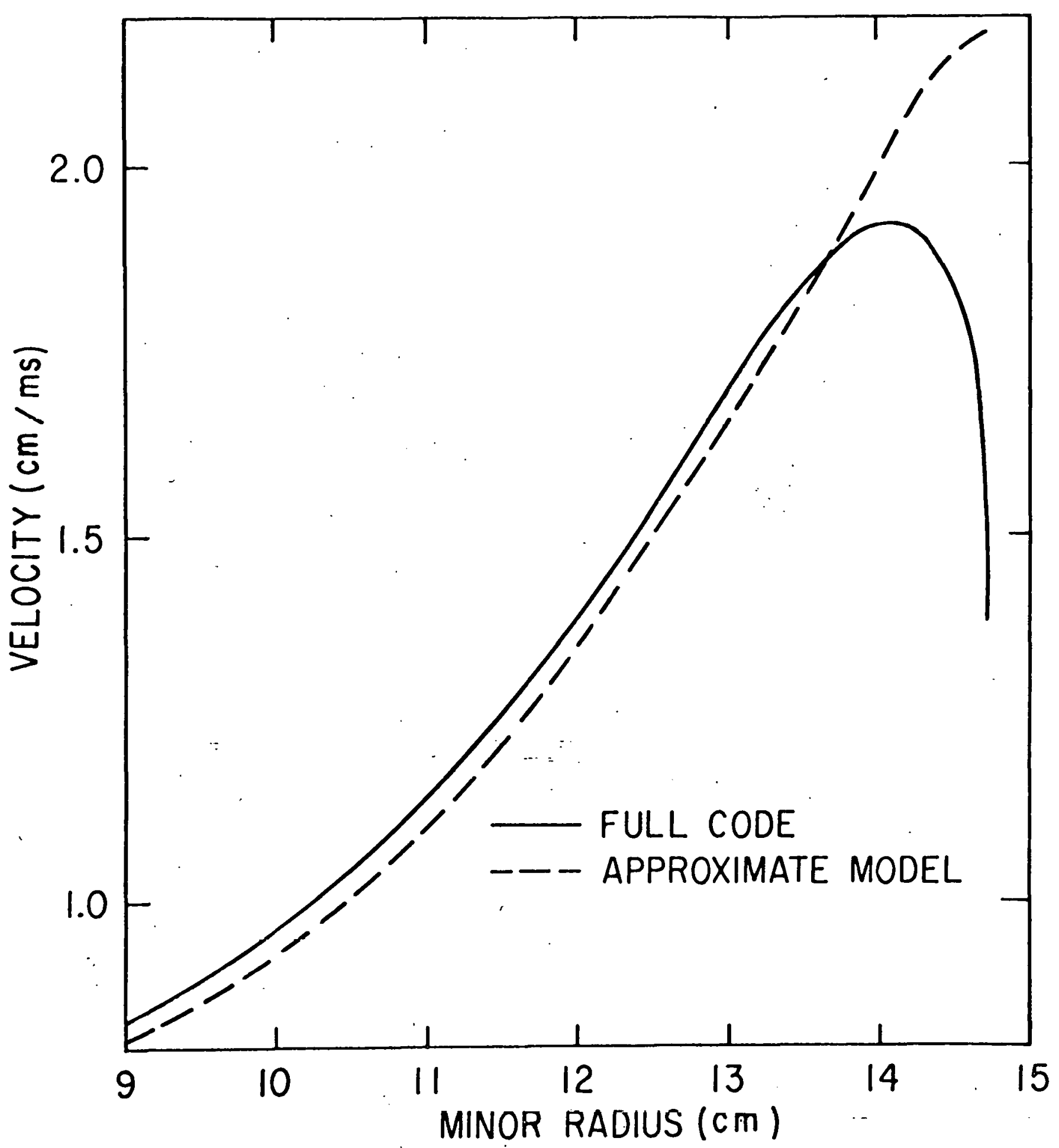

763390

Fig. 5. (b) Comparison of the diffusional velocity as a function of radius from the full code with that of the approximate model (Eq. 14). 


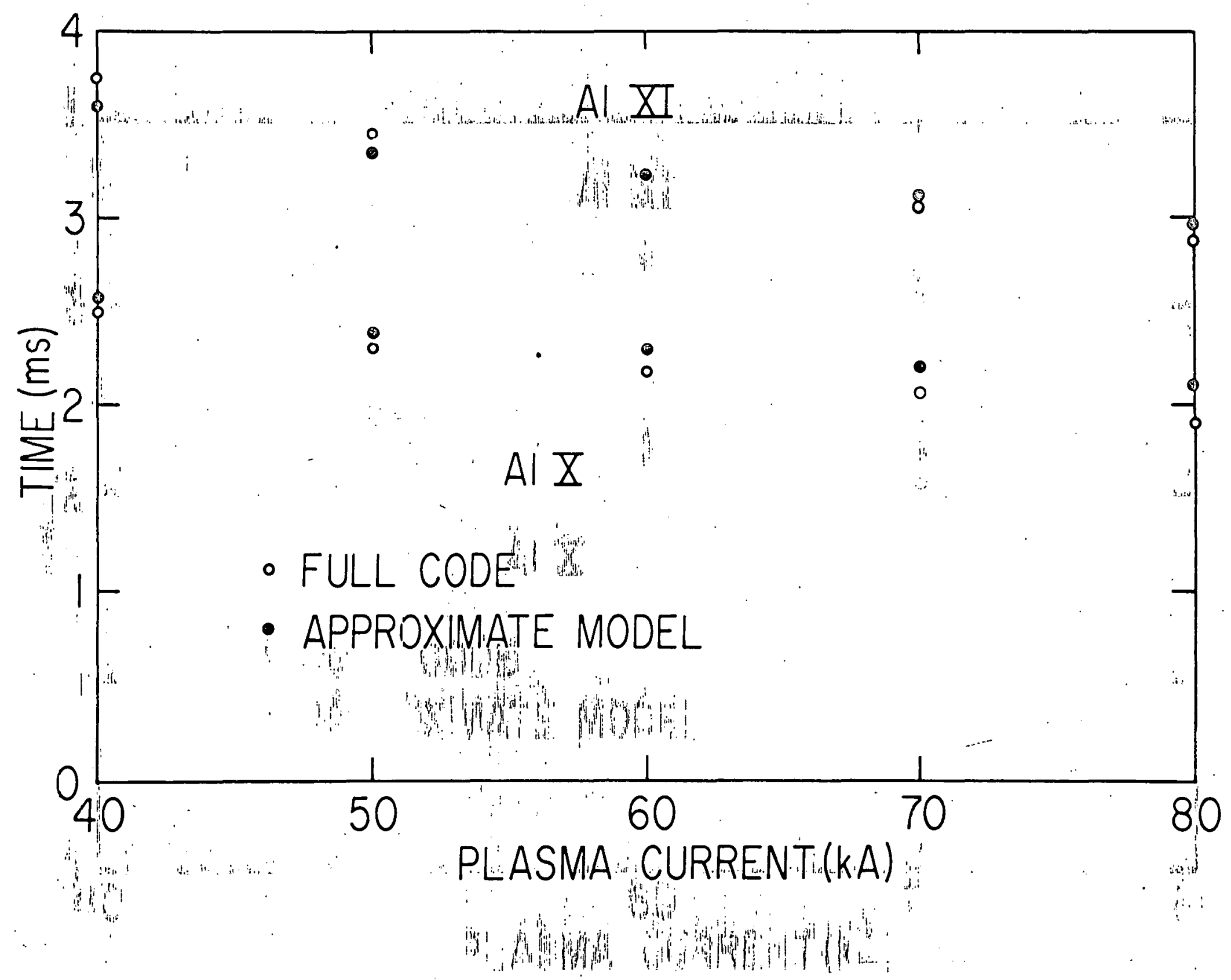

Fig. 6. Comparison of the approximate model predictions for $t_{X}$ and $t_{X I}$ with those of the full code. 


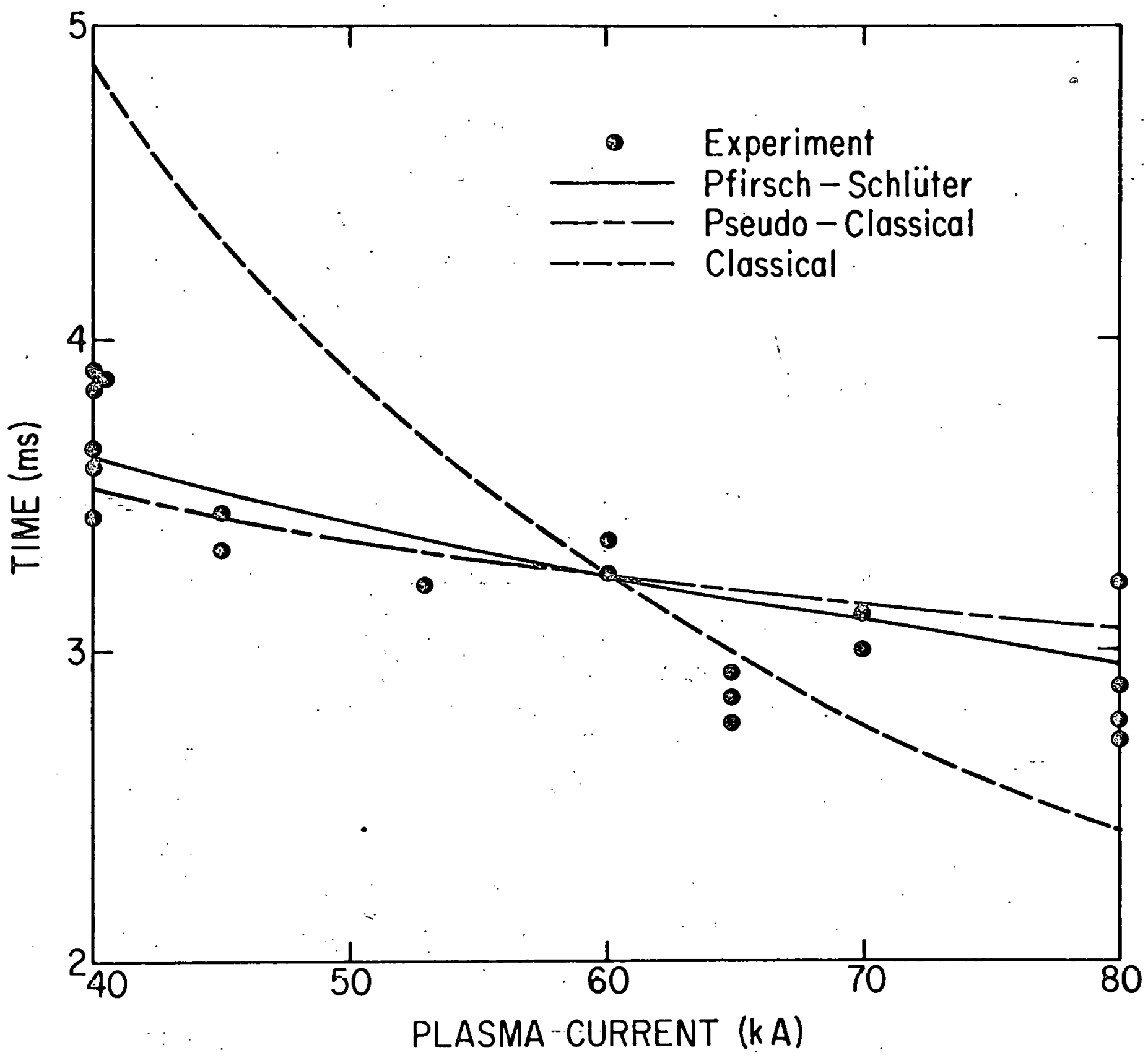

Fig. 7. Comparison with the experimental data of three different ${ }^{-}$diffusion theory predictions using the approximate model. 


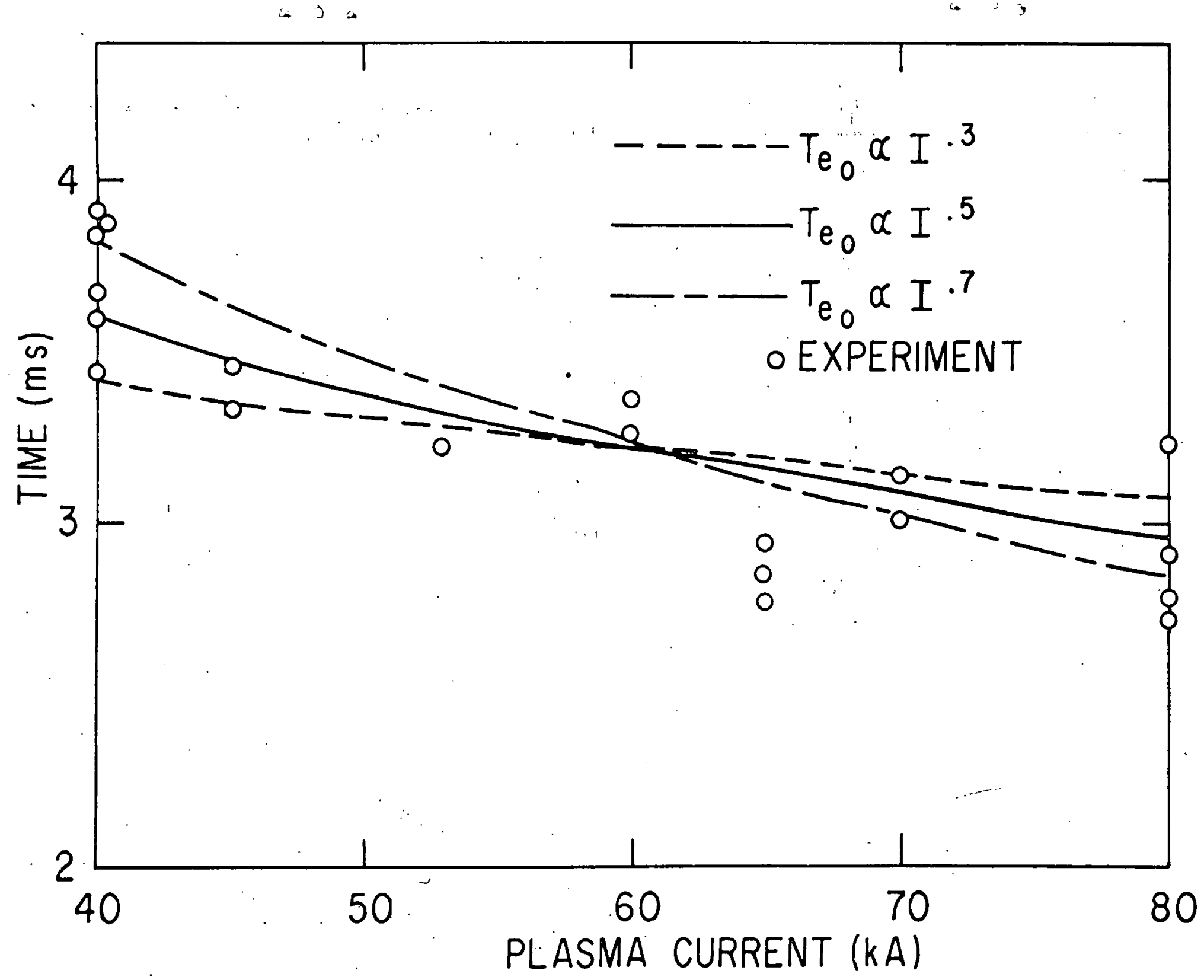

763396

Fig. 8. (a) Comparisons of $t_{X I}$ vs. $I_{p}$ using the approximate model assúming $\mathrm{T}_{\mathrm{e}_{0}} \propto \mathrm{I}_{\mathrm{p}} \cdot 3, \mathrm{I}_{\mathrm{p}} \cdot 5$ and $\mathrm{I}_{\mathrm{p}} \cdot 7$ 


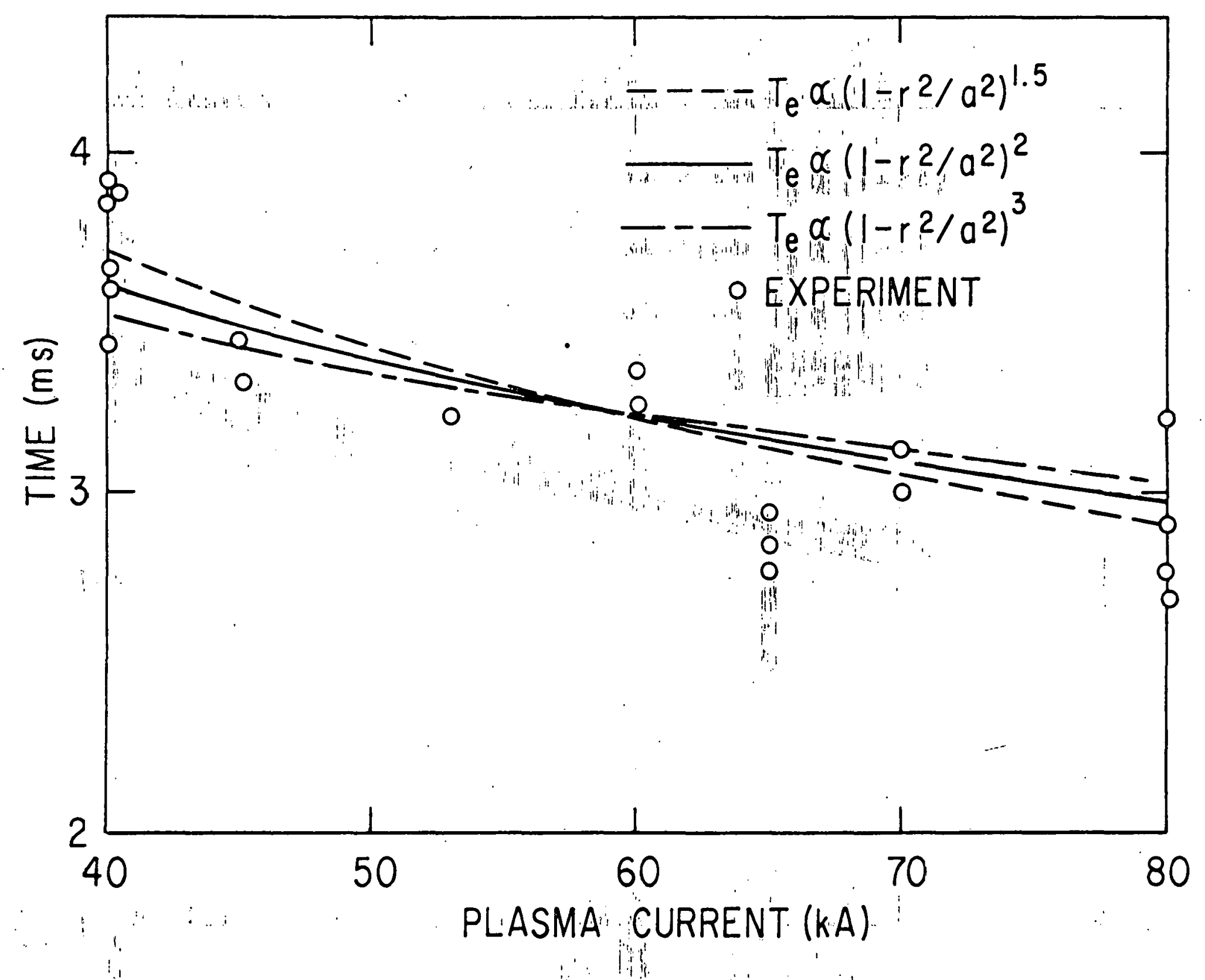

Fig. 8. (b) Comparison of txI vs. Ip using the approximate model assuming $\mathrm{Ie}_{e}\left(1-\mathrm{r}^{2} / \mathrm{a}^{2}\right)^{1.5},\left(1-\mathrm{r}^{2} / \mathrm{a}^{2} \mathrm{p}^{2}\right.$, and $\left(1-\mathrm{r}^{2} / \mathrm{a}^{2}\right)^{3}$. 


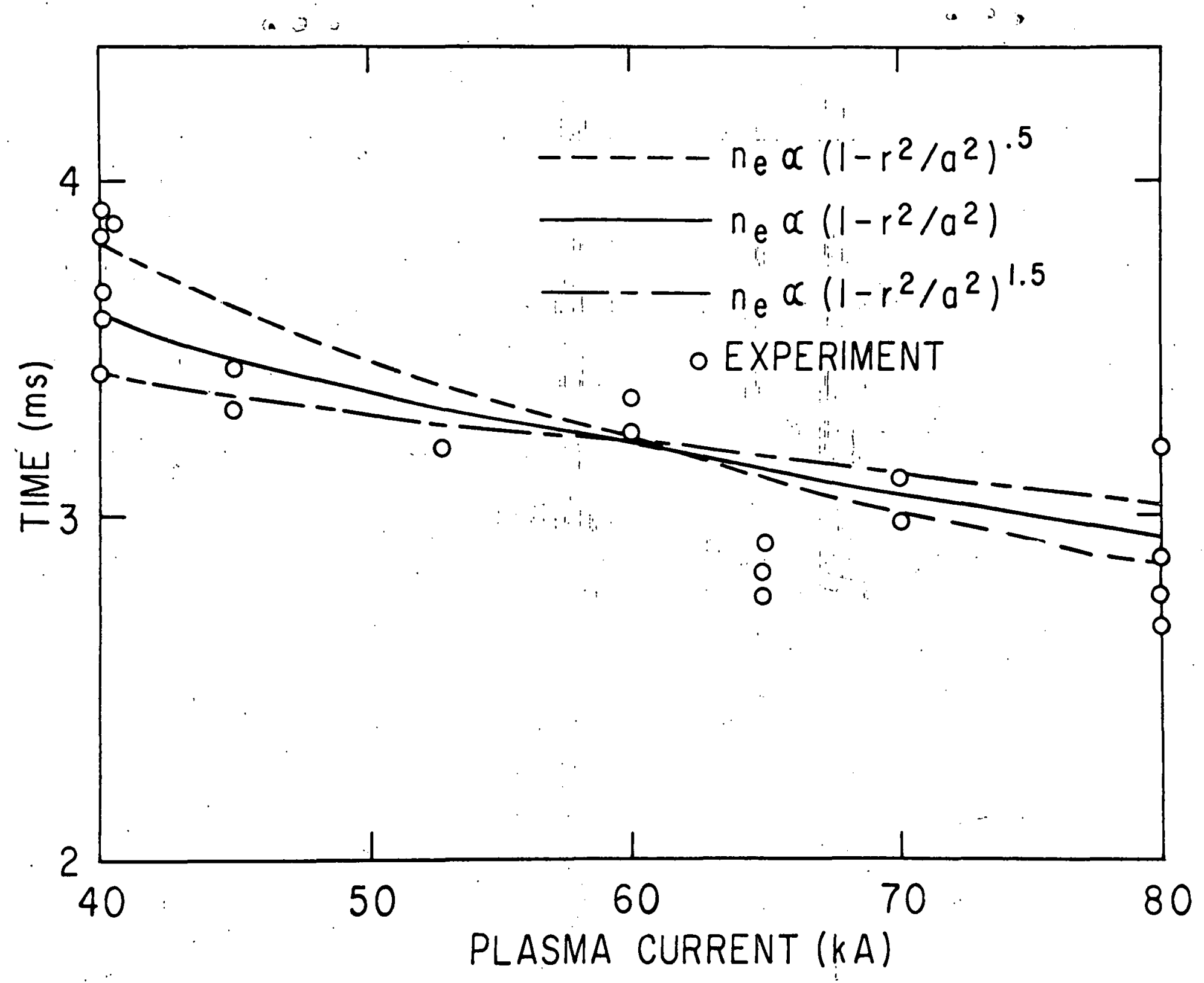
model assuming $n_{e} \propto\left(1-r^{2} / a^{2}\right) 1 / 2,\left(1-r^{2} / a^{2}\right)$, and $\left(1-r^{2} / a^{2}\right) 1.5$. 